Sánchez Fernández, M., Fragoso Campón, L., Gutiérrez Gallego J. A., Quirós Rosado E. (2017): "Caída de la demanda con la distancia en el uso del transporte público: el caso del acceso al campus universitario de Cáceres", GeoFocus (Artículos), $n^{o}$ 20, p. 141-163. ISSN: 1578-5157 http://dx.doi.org/10.21138/GF.563

\title{
CAÍDA DE LA DEMANDA CON LA DISTANCIA EN EL USO DEL TRANSPORTE PÚBLICO: EL CASO DEL ACCESO AL CAMPUS UNIVERSITARIO DE CÁCERES
}

\author{
MANUEL SÁNCHEZ FERNÁNDEZ ${ }^{1}$, LAURA FRAGOSO CAMPÓN ${ }^{1}$, JOSÉ \\ ANTONIO GUTIÉRREZ GALLEGO ${ }^{1}$, ELIA QUIRÓS ROSADO ${ }^{1}$ \\ ${ }^{1}$ Escuela Politécnica de Cáceres, Universidad de Universidad de Extremadura, Avda. \\ Universidad s/n, 10003, Cáceres. \\ msf@unex.es, laurafragoso@unex.es, jagutier@unex.es, equiros@unex.es
}

\section{RESUMEN}

Un aspecto importante a la hora de analizar la empleabilidad de un determinado servicio es la accesibilidad que tiene para los potenciales usuarios. Accesibilidad que viene determinada por el coste que tiene el acceso al servicio. Si se tiene en cuenta el acceso a un modo de transporte, generalmente se suele considerar el tiempo que se tarda en alcanzar el servicio. El concepto de caída de la demanda con la distancia (distance decay) hace referencia precisamente a la relación antedicha, es decir, relaciona el tiempo de acceso y probabilidad de uso de cualquier medio de transporte. En este artículo se desarrolla una completa metodología que permite estimar la caída de la demanda con la distancia en el empleo de autobús urbano en la ciudad de Cáceres. Bajo dos posibles escenarios de acceso a las paradas de autobús se han obtenido las funciones de la caída de la demanda y las distancias límite de uso del servicio. Mostrándose que dicho límite no sólo está influido por la distancia más corta a la parada.

Palabras Clave: accesibilidad, caída de la demanda con la distancia, función Tanner, modelos de transporte.

\section{DISTANCE DECAY IN THE USE OF PUBLIC TRANSPORT. A CASE OF STUDY: ACCESS TO THE UNIVERSITY CAMPUS OF CÁCERES.}

\section{ABSTRACT}

An important aspect when analyzing the usage of a given service is the accessibility that it has for the potential users. This Accessibility is determined by the cost of having access to the service. Considering the access to a mode of transport, it is usually estimated as the time it takes to reach the service. The concept of distance decay reflects precisely the above mentioned relation. In other words, it relates the access time and the probability of using any mode of transport. In this work a complete methodology, that allows estimating the decay distance for urban bus use in the city of Cáceres, is developed. Two possible scenarios of accessing to bus stops have been studied, obtaining for each case the function of decay and the limit distance of 
Sánchez Fernández, M., Fragoso Campón, L., Gutiérrez Gallego J. A., Quirós Rosado E. (2017): "Caída de la demanda con la distancia en el uso del transporte público: el caso del acceso al campus universitario de Cáceres", GeoFocus (Artículos), $n^{o}$ 20, p. 141-163. ISSN: 1578-5157 http://dx.doi.org/10.21138/GF.563

using the service. The results show that this limit is not only influenced by the shorter distance to the stop.

Key Words: accessibility study, decay distance, Tanner function, transports models.

\section{Introducción.}

La aparición del automóvil y su progresivo empleo generalizado a lo largo del siglo XX ha provocado cambios en los modos de desplazamientos habituales (Muñoz 2006, García Palomares y Gutiérrez Puebla 2007, Jensen 2008), donde los modos motorizados se han impuesto sobre el resto de modos. La facilidad que proveen los desplazamientos basados en sistemas de transporte motorizados ha contribuido a la dispersión de las distancias entre el centro y la periferia de las ciudades, pasando del modelo de ciudad concentrada al modelo de ciudad dispersa. El principal actor en este modelo de dispersión urbana es el vehículo privado, que asume un claro protagonismo en los desplazamientos obligados (Awad 2015). Este patrón de comportamiento conlleva evidentes costes ambientales, sociales y económicos (Díaz Muñoz et al. 2007), y constituye por tanto una movilidad de difícil sostenibilidad (Cardozo et al. 2010).

La movilidad de los habitantes de una ciudad está condicionada por el planeamiento urbano y las infraestructuras de transportes. En la ciudad de Cáceres, caracterizada por un planeamiento urbano disperso y una abrupta orografía, es imprescindible el empleo de modos de transportes motorizados para realizar los desplazamientos cotidianos. De entre dichos desplazamientos, cabe destacar los denominados movimientos obligados, es decir, aquellos que se realizan por motivos de estudio o trabajo (Ortuzar y Willumsen 2008). La característica de que este tipo de movimientos están focalizados hacia un mismo punto de destino, que a su vez alberga un gran número de trabajadores o usuarios, es la idónea para el sistema de transporte público. De ese modo, la estimación del número de desplazamientos, así como los lugares de origen y destino, facilita la planificación efectiva de un sistema que dé soporte a las necesidades creadas por los desplazamientos obligados (Jaraíz Cabanillas et al. 2013).

La accesibilidad y la demanda potencial son factores determinantes en el diseño de un sistema de transporte público eficiente (Gutiérrez Puebla et al. 2008). En ese sentido, resulta interesante estudiar la accesibilidad al transporte público, entendida como la facilidad de alcanzar un punto de acceso desde el lugar de origen del viaje. Esta cuestión adquiere cada vez mayor relevancia, ya que históricamente, en la planificación del transporte público, se le ha prestado mucha más atención al propio viaje que al recorrido hasta las paradas y estaciones (Gutiérrez Puebla et al. 2008). Al mismo tiempo, es necesario considerar que el coste al servicio supone un menor uso potencial (Cardozo et al. 2010).

Dentro del contexto urbano de la ciudad de Cáceres, este trabajo pretende cumplir dos objetivos: (i) caracterizar a los usuarios del servicio del autobús urbano para acceder al campus universitario de Cáceres; (ii) analizar la caída de la demanda con la distancia en el uso del transporte público para acceder al campus universitario, atendiendo a la proximidad de la residencia de los usuarios del servicio a las paradas de las líneas de autobús. 
Sánchez Fernández, M., Fragoso Campón, L., Gutiérrez Gallego J. A., Quirós Rosado E. (2017): "Caída de la demanda con la distancia en el uso del transporte público: el caso del acceso al campus universitario de Cáceres", GeoFocus (Artículos), $n^{o}$ 20, p. 141-163. ISSN: 1578-5157 http://dx.doi.org/10.21138/GF.563

Se considera relevante para la planificación del transporte urbano conocer los usuarios potenciales, así como la capilaridad del servicio basada en el gradiente de la distancia en función del lugar de residencia de los mismos.

El estudio se organiza en cinco apartados. Tras la presente introducción se expone el estado de la cuestión. Posteriormente se exponen los datos y metodología utilizada basada en el uso de Sistemas de Información Geográfica y análisis estadístico. En el cuarto apartado se exponen los resultados, y por último, tras la discusión, se presentan las conclusiones finales.

\section{Estado de la cuestión}

En los últimos tiempos el significado de las palabras eficiencia y sostenibilidad ha cobrado protagonismo a la hora de estudiar el comportamiento de los transportes públicos. Se pretende que el comportamiento del sistema de transporte se adapte al máximo a la demanda del mismo. Mediante Sistemas de Información Geográfica (SIG) es posible estimar el área de utilidad que un punto de acceso (parada) a un sistema de transporte proyectado sobre el viario de un municipio (Pitarch Garrido 2015). En los estudios de demanda del transporte, el uso de los SIG, es una herramienta básica y esencial para analizar la movilidad entre dos o más puntos y los factores que influyen en la misma, así como facilitar la representación gráfica del análisis efectuado (Gutiérrez Puebla et al. 2008). Por tanto, el uso de los SIG constituye la herramienta de análisis principal de este tipo de estudios, como puede comprobarse en numerosos trabajos (Mora Aliseda et al. 2003, Cardozo et al. 2010, Gutiérrez et al. 2010, Rodríguez Díaz 2011, García-Palomares et al. 2013, Pitarch Garrido 2015).

Existen distintos modelos de predicción de la demanda del transporte público, como el formulado en Zhao et al. (2003) y descrito también en Gutiérrez Puebla et al. (2008), que propone el estudio de la demanda a partir de los datos de uso de viajeros de la red de autobuses según distancia que recorren a pie desde los orígenes hasta las paradas. A través de este cálculo se establece la caída de la demanda con la distancia, que indica la tasa con la que el uso del autobús decrece con la distancia. El concepto de "caída de la demanda con la distancia" (distance decay) se define como la relación entre la probabilidad de uso del servicio y la distancia al mismo. Este concepto se utiliza para determinar el área de influencia de las paradas, como puede evidenciarse en distintos estudios (Mora Aliseda et al. 2003, Gutiérrez Puebla et al. 2008, García-Palomares et al. 2013, Martínez y Viegas 2013).

En los últimos años, se han propuesto distintos planteamientos para evaluar la caída de la demanda con la distancia. Así, en Martínez y Viegas (2013) se reflexiona sobre las formulaciones tradicionales, indicando que las funciones de uso generalizado en los modelos gravitatorios son las de tipo exponential y power, señalando también la aplicación de otras formulaciones más recientes como la función de Tanner (Ortuzar y Willumsen 2008) y la función Box-Cox (Tiefelsdorf 2003). En el trabajo señalado de Martínez y Viegas (2013) se propone además el uso de una nueva función del tipo logistic family basada en la función de Richards, concluyendo que esta propuesta se ajusta mejor a los datos empíricos especialmente en distancias cortas, descartando las del tipo exponential y power por no ajustarse a la nube de puntos de los resultados obtenidos empíricamente.

El área de influencia de un servicio de transporte público en la ciudad queda condicionada por los puntos de acceso al mismo. Por ello, cuando se estudia la caída de la 
Sánchez Fernández, M., Fragoso Campón, L., Gutiérrez Gallego J. A., Quirós Rosado E. (2017): "Caída de la demanda con la distancia en el uso del transporte público: el caso del acceso al campus universitario de Cáceres", GeoFocus (Artículos), $n^{o}$ 20, p. 141-163. ISSN: 1578-5157 http://dx.doi.org/10.21138/GF.563

demanda con la distancia, se ha de contemplar cómo un área equipotencial de uso alrededor de los puntos de acceso al servicio. En el trabajo de Quinto Bernabeu (2015), se destaca que para evaluar la influencia de un punto de acceso al transporte público se ha de limitar la distancia de influencia, pues si se usan grandes distancias los resultados obtenidos pueden quedar distorsionados. Esto es, a grandes rasgos, limitar la distancia que el viajero está dispuesto a recorrer para poder hacer uso del sistema de transporte. En algunos estudios, fundamentalmente del ámbito anglosajón, se establece como distancia límite de uso una distancia inferior a $400 \mathrm{~m}$ (Murray 2001, Zhao et al. 2003, Cardozo et al. 2010). Sin embargo, otros estudios de ámbito nacional establecen intervalos entre 300 y 600 metros (Gutiérrez Puebla et al. 2002), incluso alcanzando la cifra de 800 metros (Cardozo et al. 2010) como límite de uso del servicio de transporte público. Según Martínez y Viegas (2013), esta distancia está condicionada también por el tipo de actividad de destino y por la frecuencia con la que el viajero realiza el trayecto. En otros trabajos se indica además que esta distancia está también condicionada por el modo de transporte, de las propias características del usuario (individual y familiares) y del entorno urbano por el que se realiza el trayecto a pie (El-Geneidy et al. 2014). En este sentido, el citado estudio de El-Geneidy et al. (2014) analiza la zona de influencia del transporte público en Montreal, concluyendo que la distancia que el usuario está dispuesto a caminar en el caso del autobús es del orden de $524 \mathrm{~m}$, ascendiendo a los $1259 \mathrm{~m}$ para el caso del acceso a tren de cercanías.

En lo que se refiere al recorrido que se realiza a pie desde el origen hasta la parada de autobús, en Carpio-Pinedo (2014) se analizan distintos factores que afectan a la elección del mismo. Esta elección, no está sólo condicionada por la distancia más corta desde el origen a la parada, sino por otras variables, como pueden ser: la dificultad o tortuosidad del recorrido, la sensación de seguridad de la parada en la que se ha de permanecer hasta la llegada del autobús (zonas con afluencia de más viajeros, zonas de exclusión social, etc.), o incluso que el viajero esté dispuesto a caminar mayor distancia si con ello obtiene algún tipo de beneficio que lo compense.

Por otro lado, existen trabajos que basan el estudio de uso del transporte público en la impedancia de la distancia determinándose la importancia de ésta como condicionante en la decisión del usuario. En Jaraíz Cabanillas et al. (2015) se evalúa la tipología de los usuarios del aeropuerto de Badajoz, que se encuentra situado a $14 \mathrm{~km}$ de la ciudad, estudiando el uso del servicio en función de la distancia existente entre el aeropuerto y la residencia del usuario. El trabajo se desarrolla a partir de encuestas realizadas directamente a usuarios que se encuentran en el aeropuerto. En este trabajo se valora el factor del uso del aeropuerto a partir de la función obtenida para caída de la demanda con la distancia. Para el ajuste de la relación entre tiempo y uso potencial los autores utilizan cuatro funciones: regresión lineal, exponencial, potencial y Tanner, resultando esté último el que mejor ajuste presentó.

Atendiendo a los trabajos antedichos de Carpio-Pinedo (2014) y Jaraíz Cabanillas et al. (2015), parece lógico diferenciar la tipología de uso que pueda tener un servicio público de largo recorrido, como son el tren, el autobús de línea o ruta o el avión, de servicios de transporte público puramente urbano. En el presente trabajo se analiza el uso potencial del transporte público por las personas que acceden habitualmente al campus universitario de Cáceres, atendiendo a la proximidad entre las paradas de autobús y la residencia de los potenciales usuarios. En este sentido, son varios los estudios previos que han analizado y caracterizado la accesibilidad a distintos campus de la Universidad de Extremadura (Alfonso-Torreño 2013, 
Sánchez Fernández, M., Fragoso Campón, L., Gutiérrez Gallego J. A., Quirós Rosado E. (2017): "Caída de la demanda con la distancia en el uso del transporte público: el caso del acceso al campus universitario de Cáceres", GeoFocus (Artículos), $n^{\circ}$ 20, p. 141-163. ISSN: 1578-5157 http://dx.doi.org/10.21138/GF.563

Berrocal Nieto y Ruiz Labrador 2013, Gutiérrez Gallego y Jaraíz Cabanillas 2013, Jaraíz Cabanillas et al. 2013, Navarro Grueso 2013).

En Alfonso-Torreño (2013) se analiza el comportamiento del tráfico que accede al campus de Cáceres a partir de aforos realizados para el trabajo y encuestas de interceptación a los usuarios del campus universitario. El cálculo realizado analiza el tiempo de recorrido necesario para poder desplazarse desde el centro geométrico de las distintas barriadas al campus mediante el uso del transporte público y vehículo propio. De las encuestas se obtiene que el $55 \%$ de los usuarios utilizan el vehículo privado frente al $40 \%$ que utilizan el transporte público como medio de transporte al campus. En cuanto a un análisis por barriadas, las más cercanas a las paradas de las líneas, que permiten el viaje directo al campus, son las que mayor uso del transporte público presentan. En un modelo de transporte en el que el vehículo privado es el principal medio de transporte, el número de aparcamientos disponibles presenta un grave problema. En Navarro Grueso (2013) se realiza un análisis del uso de los estacionamientos en el campus universitario de la ciudad de Cáceres. En este caso encuestas realizadas confirmaron el dato ya señalado que el $50 \%$ de los usuarios accede al campus vía vehículo privado, determinándose además una tasa de ocupación de vehículo en todos los casos inferior a 1,50 pasajeros/vehículo excepto en el caso de los alumnos de grado en los se halla un valor de 1,99 pasajeros/vehículo. Por otro lado, en Gutiérrez Gallego y Jaraíz Cabanillas (2013), se realiza una aproximación al Plan de Movilidad Sostenible del Campus Universitario de Cáceres de la Universidad de Extremadura (UEX), en él se constata el $41 \%$ de movilidad universitaria accede al campus mediante autobús.

Existen trabajos de la misma naturaleza en otros entornos, como el de Jaraíz Cabanillas et al. (2013) en el cual se estudia la movilidad en los desplazamientos dados para los accesos de centros educativos en la ciudad de Mérida. El estudio se realiza por medio de charlas educativas a escolares separados por nivel educativo y mediante encuestas a estos mismos escolares. El estudio revela que el acceso a los colegios emeritenses es realizado casi por igual mediante vehículo privado como a pie. Finalmente, cabe destacar el trabajo de Berrocal Nieto y Ruiz Labrador (2013) que analiza la accesibilidad absoluta que presenta el transporte público en la misma ciudad de Mérida, con el fin de poder determinar que líneas o paradas pueden estar infrautilizadas, así como áreas urbanas que se encuentren en zonas de accesibilidad baja. A partir de la red viaria de la ciudad, las paradas de autobús y la distribución de la población según la ubicación de portales y habitantes por portal, se utiliza el indicador de accesibilidad absoluta para el estudio del potencial de uso del transporte público en Mérida.

\section{Materiales, datos y métodos}

\subsection{El caso de estudio}

Cáceres comienza a ser una ciudad universitaria en los años 70, y por aquel entonces, la universidad se situaba en edificaciones históricas del casco antiguo, fusionándose con la ciudad y produciéndose una integración que le daba dinamismo y vida. A medida que se pluralizaron los estudios universitarios, los edificios históricos dónde se emplazaban las distintas escuelas universitarias y facultades comenzaron a quedarse pequeñas (Campesino Fernández y Salcedo Hernández 2007), lo cual produjo una reubicación de las instalaciones hacia lo que ahora conocemos como campus universitario, que está situado al este de la ciudad, a unos 4,5 km del centro urbano (Gutiérrez Gallego y Jaraíz Cabanillas 2013). La distancia, y orografía de la 


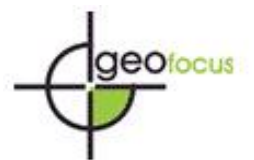

Revista Internacional de Ciencia y Tecnología de la Información Geográfica International Review of Geographical Information Science and Technology

Sánchez Fernández, M., Fragoso Campón, L., Gutiérrez Gallego J. A., Quirós Rosado E. (2017): "Caída de la demanda con la distancia en el uso del transporte público: el caso del acceso al campus universitario de Cáceres", GeoFocus (Artículos), $n^{o}$ 20, p. 141-163. ISSN: 1578-5157 http://dx.doi.org/10.21138/GF.563

ciudad (entre el centro de la ciudad y el campus existen unos $80 \mathrm{~m}$ de desnivel), son los aspectos que más dificultan la accesibilidad de la comunidad universitaria al campus. Este hecho se traduce en que el acceso al campus se realice mediante el empleo de transportes colectivos o a través del vehículo privado, siendo residual el empleo de modos no motorizados.

i. Delimitación espacial.

La Figura 1 muestra la ubicación del campus universitario situado en una de las salidas de la ciudad en la $\mathrm{N}-521$ a $4,5 \mathrm{~km}$ del centro urbano.
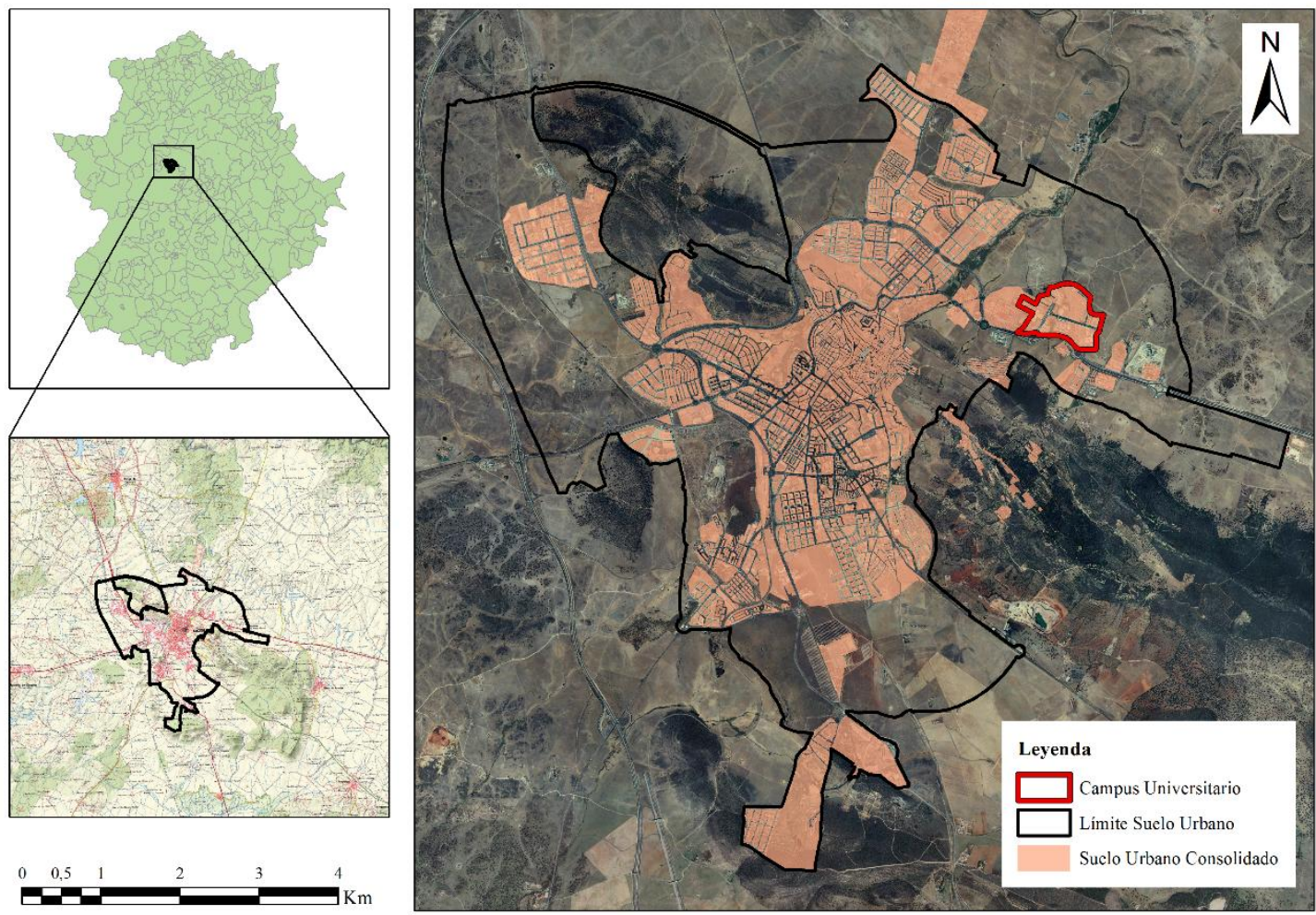

\section{Figura 1. Ubicación del campus universitario de Cáceres dentro del conjunto} urbano.

Elaboración propia utilizando la base cartográfica del Instituto Geográfico Nacional (PNOA y Cartografía Ráster).

La conexión entre campus y ciudad, mediante transporte público, se realiza por medio de tres líneas de autobús urbano (Figura 2), que acceden al campus por la N-521, vía principal de entrada al mismo. Las tres líneas se denominan: línea campus (LC) y refuerzo campus (RC), exclusivas para dar servicio al campus y línea 3 (L3), cuyo servicio no es exclusivo del mismo. Estas tres líneas de autobús cuentan con aproximadamente 10 kilómetros de recorrido cada una de ellas, coincidente además en el $90 \%$ de su trayecto. 
Sánchez Fernández, M., Fragoso Campón, L., Gutiérrez Gallego J. A., Quirós Rosado E. (2017): "Caída de la demanda con la distancia en el uso del transporte público: el caso del acceso al campus universitario de Cáceres", GeoFocus (Artículos), $n^{\circ}$ 20, p. 141-163. ISSN: 1578-5157 http://dx.doi.org/10.21138/GF.563

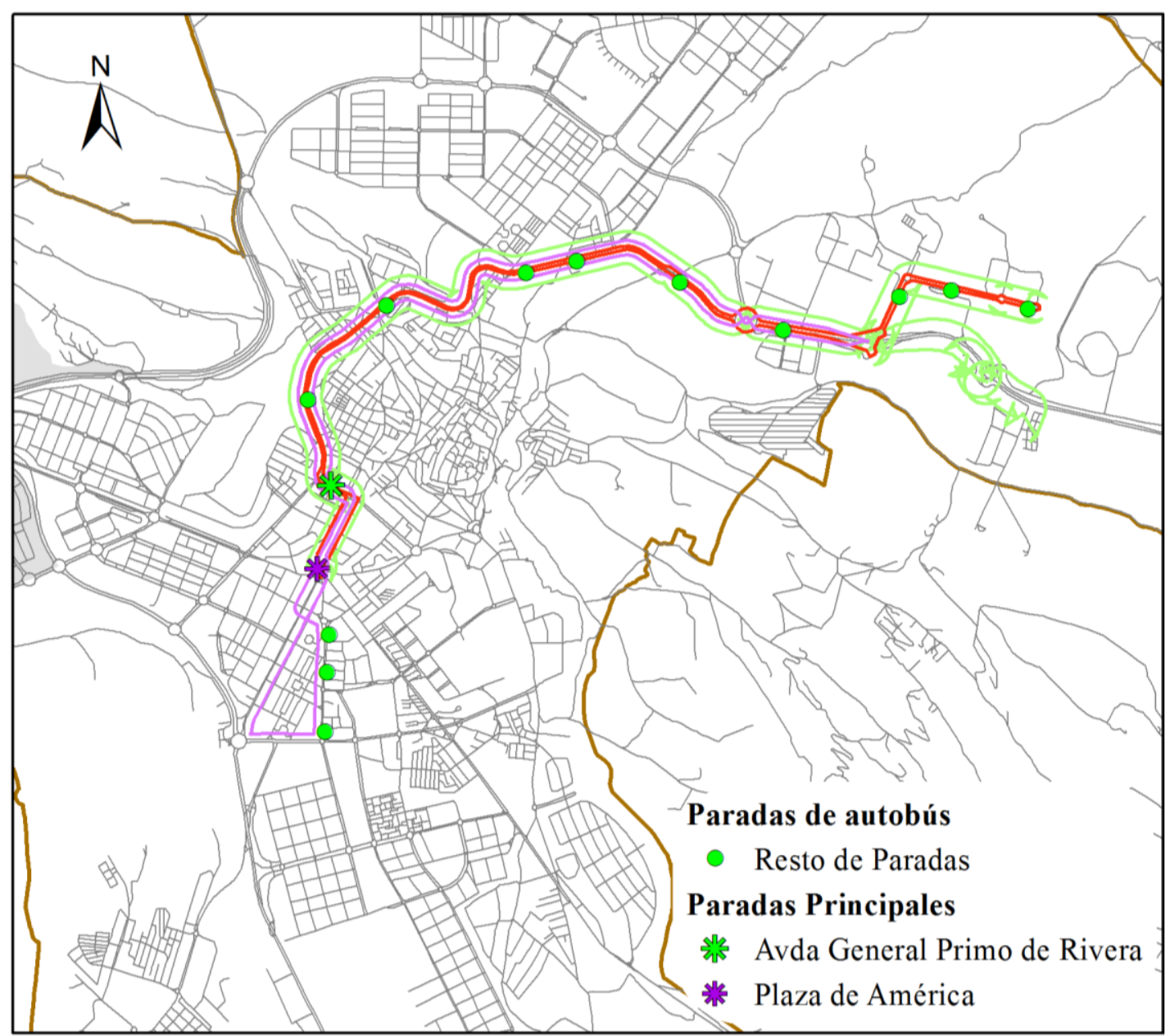

Leyenda

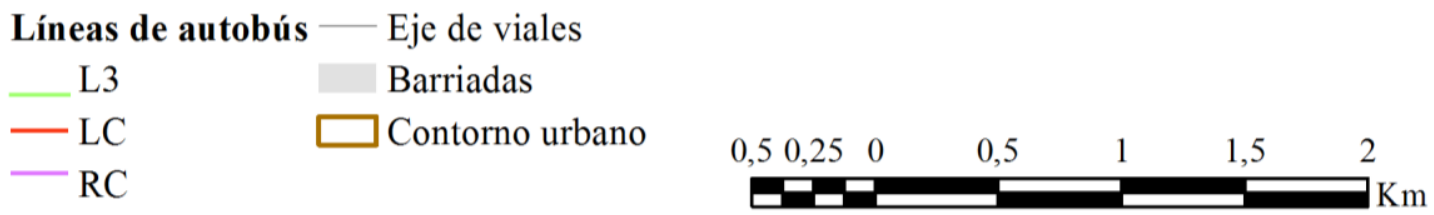

Figura 2. Líneas de autobús que conectan el centro urbano de Cáceres con el campus universitario.

Elaboración propia utilizando la base cartográfica de Cartociudad y SIG Cáceres

ii. Delimitación temporal

El presente estudio cuenta con la elaboración de una encuesta de opinión dirigida a los distintos usuarios del transporte público, que acceden habitualmente al campus universitario de Cáceres a través de autobús urbano, realizada en el mes de abril de 2015.

Por otro lado, los datos de población utilizados corresponden al padrón de habitantes del 2014 (a fecha de 1 de enero de 2015) publicado por el Instituto Nacional de Estadística. 
Sánchez Fernández, M., Fragoso Campón, L., Gutiérrez Gallego J. A., Quirós Rosado E. (2017): "Caída de la demanda con la distancia en el uso del transporte público: el caso del acceso al campus universitario de Cáceres", GeoFocus (Artículos), $n^{\circ}$ 20, p. 141-163. ISSN: 1578-5157 http://dx.doi.org/10.21138/GF.563

\subsection{Los datos}

\section{i. Encuesta de Opinión}

Un dato fundamental de partida en la presente investigación ha sido la elaboración de una encuesta de opinión dirigida a los usuarios del transporte público que acceden habitualmente al campus de Cáceres. El primer paso necesario para la realización de la encuesta consistió en determinar el tamaño muestral. Así, teniendo en cuenta el número total de personas vinculadas al campus (10.821) y los estudios previos de movilidad obligada al campus de Cáceres, como el de Gutiérrez Gallego y Jaraíz Cabanillas (2013) en que se determinó que el $41 \%$ de la población utiliza el transporte público como modo de acceso habitual, ha sido posible determinar la población a encuestar. Se ha considerado en este caso un nivel de confianza del $95 \%$ y un error máximo del $5 \%$, de tal modo que según la ecuación (1) (Cochran 1990), el tamaño muestral mínimo ascendió a 351 encuestas.

De este modo y superando el tamaño mínimo muestral, se realizaron un total de 392 encuestas durante una semana completa del mes de abril de 2015 a lo largo de turnos de mañana y tarde. La información incluida en dicha encuesta correspondía, además de a la caracterización del viaje en sí, a la localización del origen de los desplazamientos y a la parada habitual que cada encuestado emplea para acudir al campus universitario.

$$
n=\frac{N z_{\propto}^{2} s^{2}}{d^{2}(N-1)+z_{\propto}^{2} s^{2}}
$$

siendo $\mathrm{N}=$ el total de población, $z_{\propto}^{2}=$ El nivel de confianza o seguridad $(1-\alpha)$. El nivel de confianza prefijado da lugar a un coeficiente $(\mathrm{z}) \alpha$. Para un nivel de seguridad del $95 \%$ $\alpha=1,96$, para un nivel de seguridad del $99 \% \alpha=2.58, s^{2}=1$ la varianza de la distribución de la variable cuantitativa que se supone existe en la población, $\mathrm{d}=$ la precisión con que se desea estimar el parámetro ( $2 \times \mathrm{d}$ es la amplitud del intervalo de confianza).

\section{ii. Aforo}

Los datos relativos al aforo de los viajes reales realizados en las líneas de autobús estudiadas han sido facilitados por la empresa concesionaria del servicio de transporte público, SUBUS. Los datos facilitados por la empresa, correspondientes a las líneas de acceso al campus (LR, RC y L3), están referidos a dos semanas estandarizadas de distinto semestre del periodo lectivo del curso 2014-2015, una en noviembre y otra en febrero. Del análisis de las cifras se obtuvo que el número de viajes totales en un día medio a lo largo de una semana tipo es de 8.028 viajes.

iii. Fuentes de datos cartográficas

Las fuentes de datos cartográficas empleadas en el estudio han sido las siguientes:

- Cartociudad: es un proyecto del Instituto Geográfico Nacional que ofrece distintos datos espaciales de cobertura nacional como son la red viaria, cartografía urbana y toponimia, códigos postales y distritos censales (Cartociudad 2015). En el presente estudio se ha usado de la red viaria y la información urbana que ofrece Cartociudad para el año 2015.

- SIG Cáceres: se trata de IDE del Ayuntamiento de Cáceres (SIG_Cáceres 2015) que facilita distintos datos entre los que destacamos, las líneas y paradas del 
Sánchez Fernández, M., Fragoso Campón, L., Gutiérrez Gallego J. A., Quirós Rosado E. (2017): "Caída de la demanda con la distancia en el uso del transporte público: el caso del acceso al campus universitario de Cáceres", GeoFocus (Artículos), $n^{o}$ 20, p. 141-163. ISSN: 1578-5157 http://dx.doi.org/10.21138/GF.563

servicio del autobús urbano que da acceso al campus universitario (capa KMZ "Bus urbano de Cáceres"), utilizadas en el presente estudio.

\section{iv. Fuentes de datos demográficas}

Las fuentes de datos demográficas consultadas para la elaboración del estudio han sido las siguientes:

- Padrón continuo por unidad poblacional del INE (INE 2015).

- Datos del padrón de habitantes por portal de la ciudad de Cáceres facilitados por el Excelentísimo Ayuntamiento de Cáceres.

- Datos del Observatorio de Indicadores elaborados por la Unidad Técnica de Evaluación y Calidad de la Universidad de Extremadura (OBIN 2015).

En cuanto a los datos demográficos, conviene subrayar que la ciudad de Cáceres cuenta con 95.855 habitantes, según el padrón del 2014 publicado por el Instituto Nacional de Estadística (INE 2015). Sin embargo, esta información global ha sido completada por los datos facilitados por el Excmo. Ayuntamiento de Cáceres que, aunque ofrece datos sensiblemente distintos, sus diferencias en cuanto a datos globales de población, representan un porcentaje despreciable (inferior al $1 \%$ en los últimos 10 años). La utilización de los datos del ayuntamiento, se ha debido a la posibilidad que éstos ofrecen para analizar la distribución espacial de la población dentro de la zona urbana, ya que los datos disponibles detallan los habitantes en cada portal.

Por otro lado, cabe destacar también que la UEx cuenta con una Unidad Técnica de Evaluación y Calidad que publica anualmente indicadores para evaluar el desarrollo de la institución. En este sentido, a través del Observatorio de Indicadores (OBIN 2015) se han determinado las personas vinculadas directamente con la UEx en cada campus. En la Tabla 1 se muestra un resumen del OBIN correspondiente a los usuarios del campus de Cáceres, agrupados según su vinculación con la UEx: alumnos de grado, máster, personal de administración y servicios (PAS), personal docente e investigador (PDI) y otros (que engloba a otras personas vinculadas con la universidad de forma indirecta como el personal de otros centros de trabajo ubicados en el campus universitario de Cáceres). Según se puede apreciar en la Tabla 1, un total de 10.281 personas se encuentran directamente vinculadas con el campus universitario de Cáceres, que supone aproximadamente un $10 \%$ de la población de la ciudad.

Tabla 1. Personal del Campus de Cáceres en el curso académico 2.014/15.

Fuente: Datos del Observatorio de Indicadores de Calidad de la Universidad de Extremadura (OBIN)

\begin{tabular}{lrrrr}
\hline Personal & Hombres & \multicolumn{1}{c}{ Mujeres } & \multicolumn{1}{c}{ Total } & $\begin{array}{r}\text { Porcentaje de } \\
\text { representación }\end{array}$ \\
\cline { 1 - 4 } Grado & 3808 & 4476 & 8284 & $80,85 \%$ \\
Máster & 327 & 419 & 746 & $7,26 \%$ \\
PAS & 112 & 102 & 214 & $2,08 \%$ \\
PDI & 475 & 279 & 754 & $7,33 \%$ \\
Otros & 141 & 142 & 283 & $2,75 \%$ \\
\hline Total & 4863 & 5418 & 10281 & \\
\cline { 1 - 3 } Total $(\%)$ & $47,30 \%$ & $52,70 \%$ & $100 \%$ & \\
\cline { 1 - 3 } & & &
\end{tabular}


Sánchez Fernández, M., Fragoso Campón, L., Gutiérrez Gallego J. A., Quirós Rosado E. (2017): "Caída de la demanda con la distancia en el uso del transporte público: el caso del acceso al campus universitario de Cáceres", GeoFocus (Artículos), $n^{\circ}$ 20, p. 141-163. ISSN: 1578-5157 http://dx.doi.org/10.21138/GF.563

\subsection{Técnicas de Análisis}

Una vez realizadas las encuestas, se procesaron los datos obtenidos de la misma, volcando la información en una base de datos de Microsoft Access, a partir de ello, en primer lugar se caracterizó al usuario de las líneas de acceso al campus. En segundo lugar, y mediante la utilización conjunta de la base de datos y un Sistema de Información Geográfica (utilizado el software ArcGIS), se georreferenciaron las ubicaciones de las viviendas de los usuarios encuestados, en base a la localización de los portales de Cartociudad (Cartociudad 2015). En algunos de los casos, hubo que completar manualmente ciertos portales que no existían en la información cartográfica descargada. Para realizar esta tarea fue necesario recurrir a las Ortofotos del Plan Nacional de Ortofotografía Aérea (PNOA) y a la información de catastro, ambas facilitadas por el Instituto Geográfico Nacional (IGN).

Como ya se ha mencionado anteriormente, en un modelo de demanda de transporte, la caída de la demanda con la distancia representa el efecto disuasivo de la distancia sobre los desplazamientos, de forma que se generan gradientes concéntricos decrecientes con la distancia (Gutiérrez Puebla et al. 2008). En nuestro caso, la caída de la demanda con la distancia en el uso del transporte público para acceder al campus universitario, en función de la accesibilidad a las paradas, se calculó a partir de la distancia desde cada punto de origen (portal de la vivienda) hasta las paradas de autobús.

En base a los resultados de la caracterización del usuario se detectó que, de las 15 paradas existentes en todo el recorrido, dos de ellas concentran más del $80 \%$ de los usuarios, que corresponden a las paradas de Plaza de América y Avda. General Primo de Rivera (Figura 2). Ante estos dos focos de atracción se consideró plantear el análisis en dos escenarios de transporte alternativos: uno considerando que el usuario utiliza la parada más próxima a su domicilio y otro suponiendo que el usuario accede al servicio desde una de las dos paradas principales, considerando en este caso que, de las dos paradas posibles, utilizará la más próxima a su domicilio.

Para obtener las distancias desde cada origen (portal) hasta cada destino (la parada de autobús) se generó una matriz $\mathrm{OD}$, que muestra la distancia o coste de recorrido entre todos los orígenes y los destinos. El coste o impedancia asignada a la red se corresponde con la longitud en metros de cada tramo. Para ello se generó la red topológica sobre el entramado viario de la ciudad. En el cálculo se obtuvo la matriz OD para cada uno de los escenarios del modelo de transporte, que considera como orígenes todos los portales, y destino las paradas de autobús.

El cálculo de la función de la caída de la demanda con la distancia se llevó a cabo, con ayuda del software R (Team 2016), y mediante un ajuste por mínimos cuadrados basado en la función Tanner (Ortuzar y Willumsen 2008, Martínez y Viegas 2013), cuya expresión general se muestra en la ecuación (2):

$$
y=x^{\beta_{1}} e^{x \beta_{2}}
$$

siendo $y$ el ratio (la probabilidad que tiene una persona ubicada en un portal de utilizar el servicio), $x$ la distancia (desde el portal hasta la parada), y por último, $\beta_{1}$ y $\beta_{2}$, que corresponden a los parámetros del ajuste. 
Sánchez Fernández, M., Fragoso Campón, L., Gutiérrez Gallego J. A., Quirós Rosado E. (2017): "Caída de la demanda con la distancia en el uso del transporte público: el caso del acceso al campus universitario de Cáceres", GeoFocus (Artículos), $n^{\circ}$ 20, p. 141-163. ISSN: 1578-5157 http://dx.doi.org/10.21138/GF.563

Para realizar el ajuste se establecieron distintos rangos de distancias, adecuándolos a cada uno de los escenarios propuestos para la formación del modelo de transporte (parada más próxima al domicilio o elección de una de las paradas principales).

Finalmente a través de la función de Tanner y los parámetros obtenidos del ajuste realizado se calculó el valor del ratio para cada uno de los portales de la ciudad. En base a las distancias desde los domicilios de los usuarios encuestados a las paradas, se obtienen: los viajes potenciales, la estimación de la población para cada uno de los intervalos y el ratio viajes/población, que representa la probabilidad de uso del servicio.

Esta acción se aplica a los dos modelos de transporte descritos. A partir del método de interpolación de la distancia inversa ponderada (IDW) se obtuvo la distribución espacial continua del ratio de toda la ciudad.

\section{Descripción y análisis de los resultados}

\subsection{Caracterización del usuario}

Atendiendo a la distribución de usuarios según el género, el usuario mayoritario del autobús corresponde a las mujeres, con un $55 \%$ del total de los usuarios encuestados. En la Figura 3 se observa que los valores recogidos en la encuesta en función al género de los usuarios son sensiblemente similares a la distribución por géneros de la población universitaria en general $( \pm 2,15 \%)$.

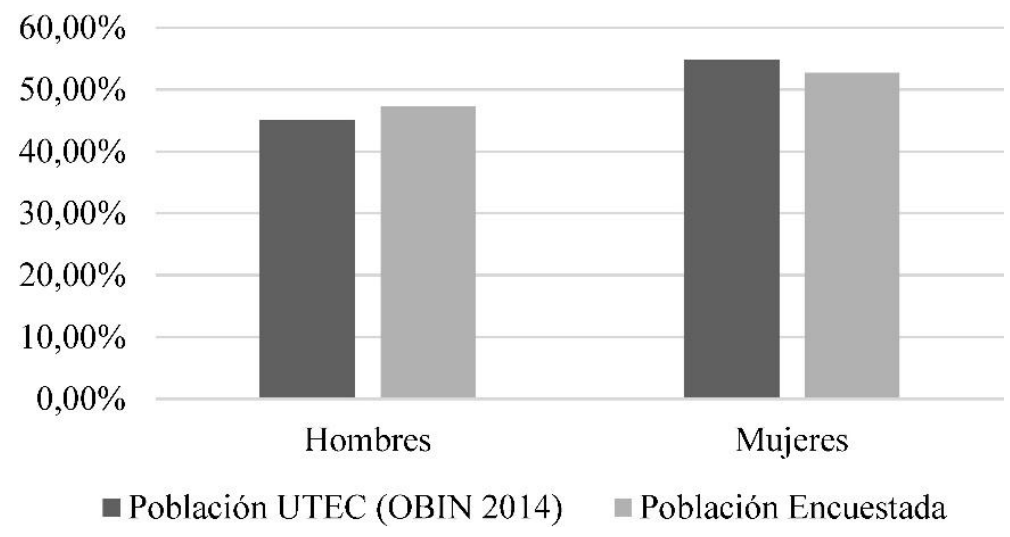

Figura 3. Distribución por géneros.

Fuente: OBIN 2014; Encuestas realizadas y elaboración propia. 
Sánchez Fernández, M., Fragoso Campón, L., Gutiérrez Gallego J. A., Quirós Rosado E. (2017): "Caída de la demanda con la distancia en el uso del transporte público: el caso del acceso al campus universitario de Cáceres", GeoFocus (Artículos), $n^{o}$ 20, p. 141-163. ISSN: 1578-5157 http://dx.doi.org/10.21138/GF.563

Con respecto a la edad del usuario, el $90 \%$ de la población encuestada es menor de 25 años, siendo el $43 \%$ menor de 20 años, y el $47 \%$ se encuentra en el rango entre 21 y 25 años. En este sentido se obtiene además que el $93 \%$ de los usuarios encuestados son estudiantes frente al $88 \%$ de representación que tienen éstos según la UTEC (Figura 4).

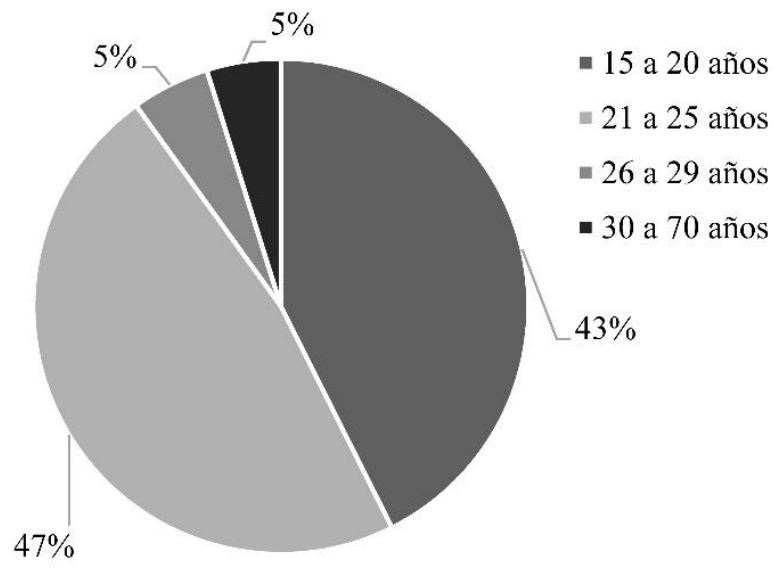

Figura 4. Distribución por edades

Datos de encuesta realizada. Elaboración propia.

Finalmente, cabe destacar que las dos paradas principales representan un punto de confluencia de barriadas residenciales estimadas céntricas en la ciudad. La atracción de las mismas sobre los usuarios de las líneas que dan acceso al campus universitario, se puede observar gráficamente en la Figura 5, en esta se muestra la distribución espacial obtenida a partir de la ubicación de los domicilios de las personas encuestadas 
Sánchez Fernández, M., Fragoso Campón, L., Gutiérrez Gallego J. A., Quirós Rosado E. (2017): "Caída de la demanda con la distancia en el uso del transporte público: el caso del acceso al campus universitario de Cáceres", GeoFocus (Artículos), $n^{\circ}$ 20, p. 141-163. ISSN: 1578-5157 http://dx.doi.org/10.21138/GF.563

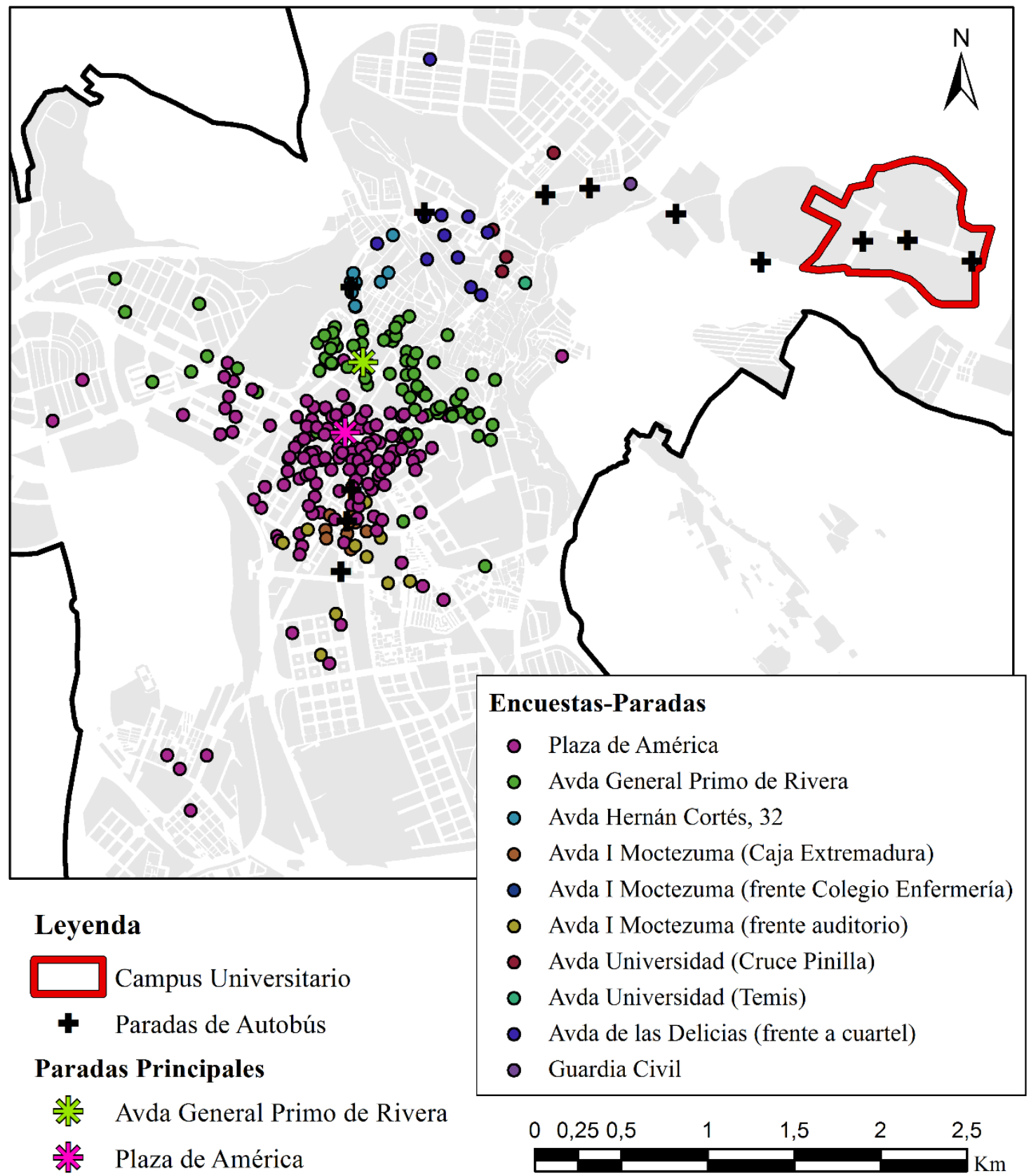

Figura 5. Distribución espacial de las viviendas de los usuarios encuestados.

\subsection{Función de la caída de la demanda con la distancia}

Se realiza el cálculo de la función de la caída de la demanda con la distancia para los dos escenarios descritos anteriormente.

i. Escenario 1- Caso de utilización de la parada más próxima al domicilio.

En el primer escenario se contempla la hipótesis de que el usuario utilizará la parada de autobús, de las líneas que dan acceso al campus, que más cerca se encuentre de su domicilio. En 
Sánchez Fernández, M., Fragoso Campón, L., Gutiérrez Gallego J. A., Quirós Rosado E. (2017): "Caída de la demanda con la distancia en el uso del transporte público: el caso del acceso al campus universitario de Cáceres", GeoFocus (Artículos), $n^{\circ}$ 20, p. 141-163. ISSN: 1578-5157 http://dx.doi.org/10.21138/GF.563

base a las distancias desde los domicilios de los usuarios encuestados a las paradas, se obtienen (Tabla 2): los viajes potenciales, la estimación de la población para cada uno de los intervalos y el ratio viajes/población, que representa la probabilidad de uso del servicio.

Tabla 2. Tabla de resultado del Escenario 1

\begin{tabular}{c|rrr}
\hline $\begin{array}{c}\text { Distancias } \\
(\mathbf{m})\end{array}$ & \multicolumn{1}{l}{ Viajes } & Población & \multicolumn{1}{c}{ Ratio } \\
\hline $0-125$ & 379,25 & 3128 & 0,121 \\
$125-250$ & 625,25 & 7816 & 0,080 \\
$250-400$ & 902,00 & 12656 & 0,071 \\
$400-600$ & 871,25 & 15578 & 0,056 \\
$600-900$ & 676,50 & 17481 & 0,039 \\
$900-1400$ & 317,75 & 15059 & 0,021 \\
$1400-1500$ & 41,00 & 2435 & 0,017 \\
$1500-2000$ & 92,25 & 9386 & 0,010 \\
$2000-2500$ & 112,75 & 12316 & 0,009 \\
\hline
\end{tabular}

Aplicando un ajuste por mínimos cuadrados a la función de Tanner, podemos extender el valor del ratio al conjunto de la población. La Figura 6 muestra el ajuste realizado, y se puede observar como la función resultante se ajusta, con un error medio cuadrático (EMC) $r^{2}=$ 0,00509535 , a todos los puntos representados.

\section{Tanner}

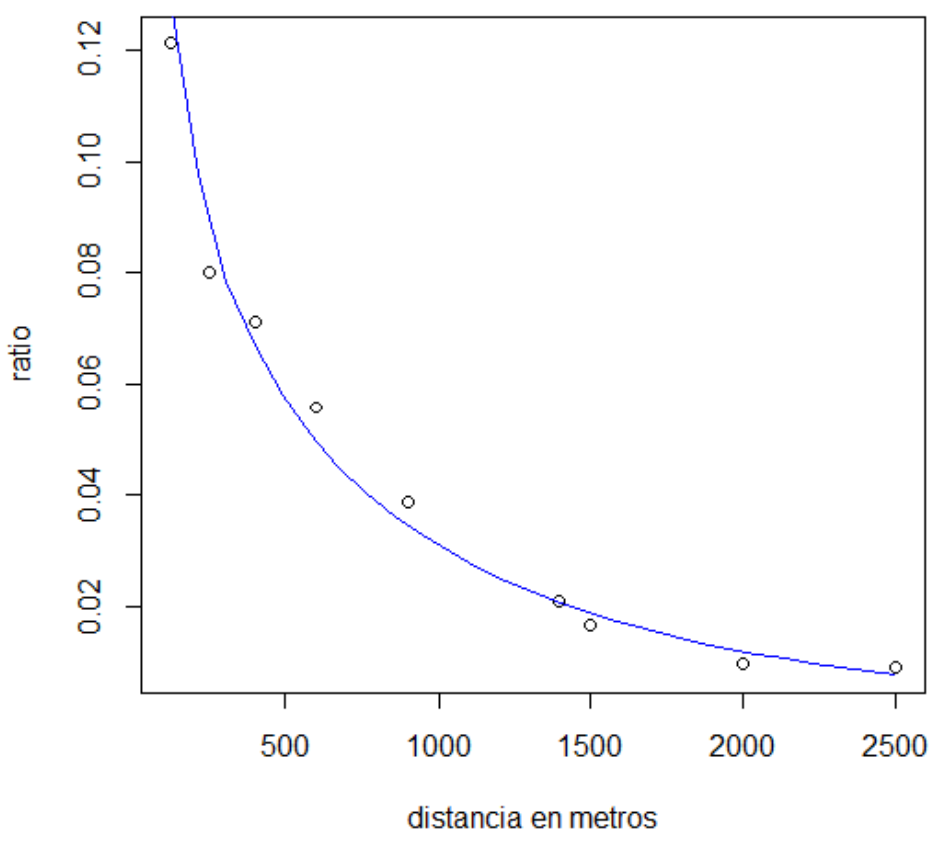

Figura 6. Ajuste de mínimos cuadrados mediante la función de Tanner para el Escenario 1. 
Sánchez Fernández, M., Fragoso Campón, L., Gutiérrez Gallego J. A., Quirós Rosado E. (2017): "Caída de la demanda con la distancia en el uso del transporte público: el caso del acceso al campus universitario de Cáceres", GeoFocus (Artículos), $n^{o}$ 20, p. 141-163. ISSN: 1578-5157 http://dx.doi.org/10.21138/GF.563

La Figura 7 representa el mapa de la caída de la demanda con la distancia obtenida para el Escenario 1 y se observa que los residentes que tiene mayor probabilidad de utilizar el servicio del autobús urbano que da acceso al campus universitario, son los más cercanos a la trayectoria del servicio que recorre las siguientes vías: Av. Isabel de Moctezuma - Av. de España - Av. General Primo de Rivera - Av. Hernán Cortés - Av. de las Delicias.



Leyenda

- Parada de autobús Probabilidad de usar el servicio

— Línea de autobús

Barriadas

(viajes/habitante)

Contorno urbano

Eje de viales
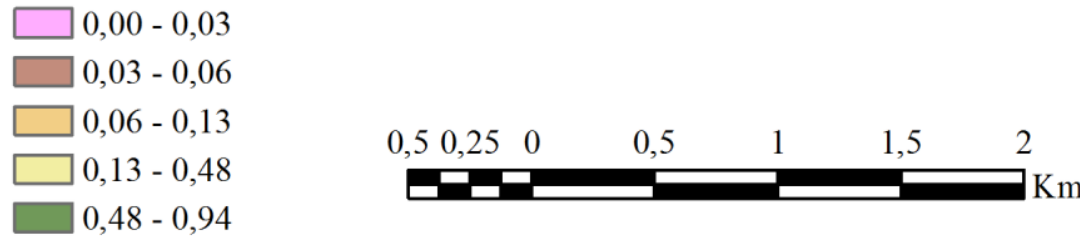

Figura 7. Caída de la demanda con la distancia en el uso del autobús para acceder al campus universitario de Cáceres en función de la accesibilidad de las paradas para el Escenario 1. 
Sánchez Fernández, M., Fragoso Campón, L., Gutiérrez Gallego J. A., Quirós Rosado E. (2017): "Caída de la demanda con la distancia en el uso del transporte público: el caso del acceso al campus universitario de Cáceres", GeoFocus (Artículos), $n^{\circ}$ 20, p. 141-163. ISSN: 1578-5157 http://dx.doi.org/10.21138/GF.563

ii. Escenario 2- Caso de utilización de la parada principal más próxima al domicilio.

En el segundo escenario se plantea la hipótesis de que el usuario utilizará, de las dos paradas de autobús principales, la que esté más cerca de su domicilio, intentado reflejar la tendencia observada a través de las encuestas, que han mostrado que las dos paradas principales son las que dan servicio a $82 \%$ de los usuarios encuestados.

Del mismo modo que el expuesto para el Escenario 1, la Tabla 3 muestra los resultados obtenidos. El ratio disminuye bruscamente a partir del cuarto intervalo $(900-1.300 \mathrm{~m})$, reduciéndose casi a la mitad respecto del intervalo anterior, es decir, respecto del tercer intervalo $(600-900 \mathrm{~m})$.

Tabla 3. Tabla de resultados del Escenario 2.

\begin{tabular}{c|rrr}
\hline $\begin{array}{c}\text { Distancias } \\
(\boldsymbol{m})\end{array}$ & \multicolumn{1}{|c}{ Viajes } & \multicolumn{1}{c}{ Población } & \multicolumn{1}{c}{ Ratio } \\
\hline $0-300$ & 1014,75 & 7102 & 0,143 \\
$300-600$ & 1424,75 & 14327 & 0,099 \\
$600-900$ & 779,00 & 14765 & 0,053 \\
$900-1300$ & 492,00 & 13925 & 0,035 \\
$1300-1900$ & 123,00 & 15405 & 0,008 \\
$1900-2200$ & 71,75 & 4824 & 0,015 \\
$2200-2500$ & 112,75 & 25507 & 0,004 \\
\hline
\end{tabular}

Obrando del mismo modo, al Escenario 2, se le aplica un ajuste por mínimos cuadrados a la función de Tanner con el que extender el valor del ratio al conjunto de la población. En la Figura 8 se observa que el ajuste realizado se adapta mejor a los cuatro primeros intervalos de distancias. El ajuste por mínimos cuadrados realizado presenta un EMC $\mathrm{r}^{2}=0,007593017$ 
Sánchez Fernández, M., Fragoso Campón, L., Gutiérrez Gallego J. A., Quirós Rosado E. (2017): "Caída de la demanda con la distancia en el uso del transporte público: el caso del acceso al campus universitario de Cáceres", GeoFocus (Artículos), $n^{o}$ 20, p. 141-163. ISSN: 1578-5157 http://dx.doi.org/10.21138/GF.563

Tanner

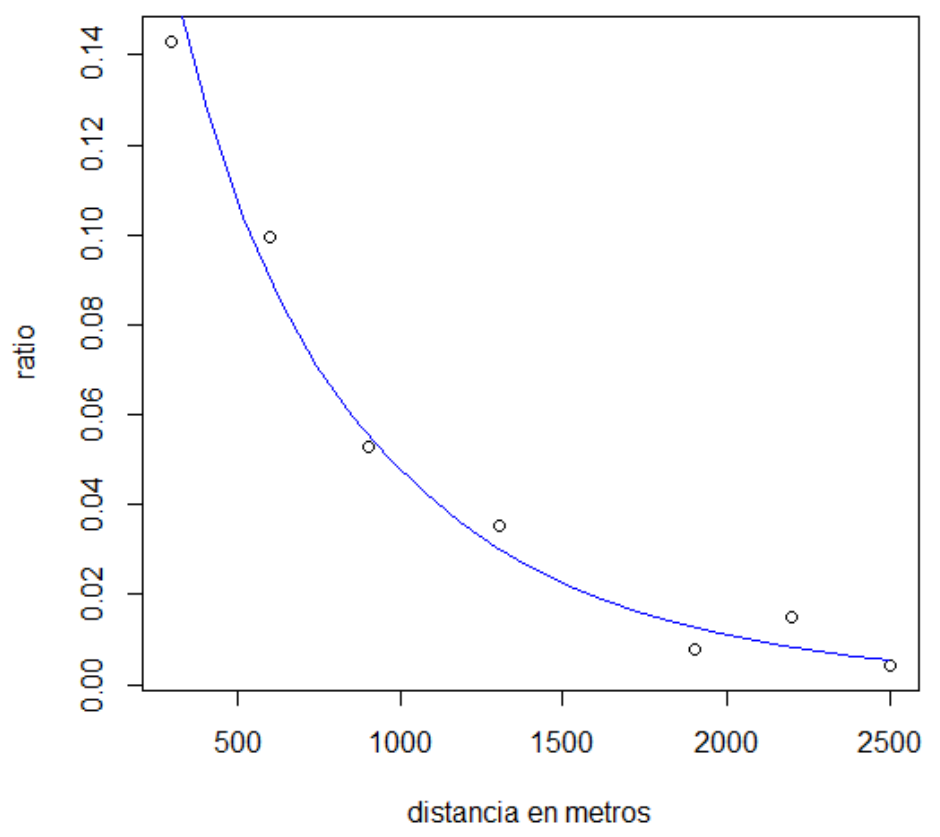

Figura 8. Ajuste de mínimos cuadrados mediante la función de Tanner para el Escenario 2.

En la Figura 9 se observa que la máxima probabilidad de que un ciudadano utilice el servicio de autobús del campus se centra en torno al Parque de Cánovas, por su ubicación entre las dos paradas estudiadas. Este escenario ordena de forma concéntrica la probabilidad de utilizar el servicio de autobús, englobando la mayor parte de la zona centro del núcleo urbano de Cáceres. 


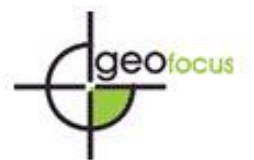

Revista Internacional de Ciencia y Tecnología de la Información Geográfica International Review of Geographical Information Science and Technology

Sánchez Fernández, M., Fragoso Campón, L., Gutiérrez Gallego J. A., Quirós Rosado E. (2017): "Caída de la demanda con la distancia en el uso del transporte público: el caso del acceso al campus universitario de Cáceres", GeoFocus (Artículos), $n^{o}$ 20, p. 141-163. ISSN: 1578-5157 http://dx.doi.org/10.21138/GF.563

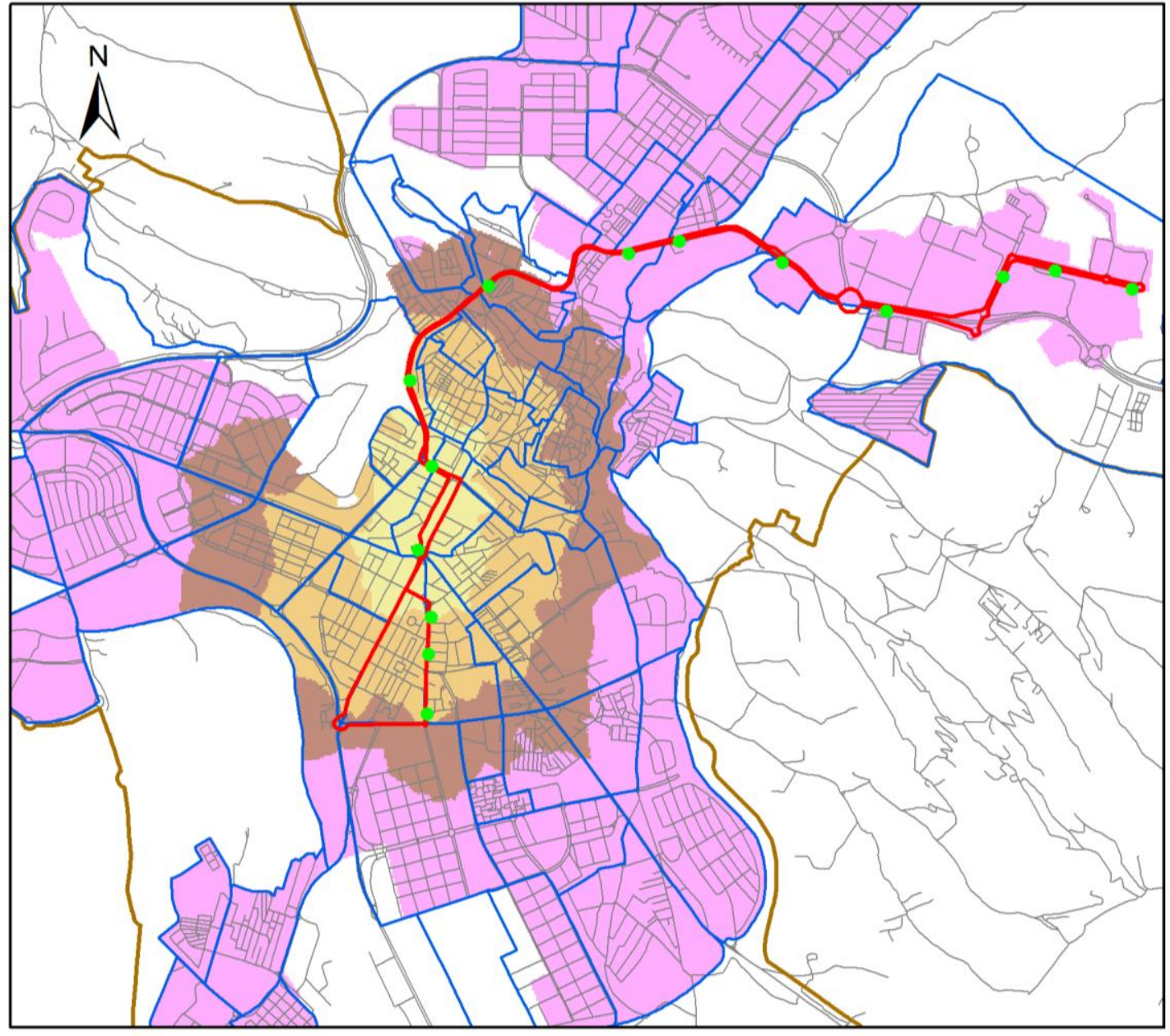

\section{Leyenda}

- Parada de autobús Probabilidad de usar el servicio

- Línea de autobús

$\square$ Barriadas

(viajes/habitante)

Contorno urbano

$\square 0,00$ - 0,03

_ Eje de viales
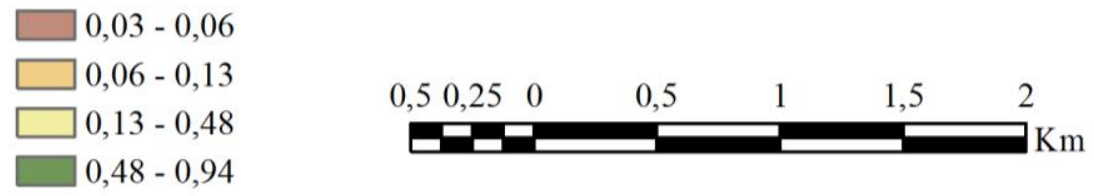

Figura 9. Caída de la demanda con la distancia en el uso del autobús para acceder al campus universitario de Cáceres en función de la accesibilidad de las paradas para el Escenario 2

El segundo escenario representa una tendencia radial de probabilidad de uso del servicio a partir de las dos paradas estudiadas. Ésta probabilidad disminuye conforme se aleja del centro del escenario establecido, que en este caso, es el centro de gravedad del entorno urbano de la ciudad, el parque de Cánovas. En este segundo escenario aparece una zona más compacta en el primer intervalo. 


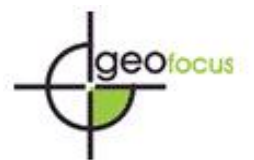

Sánchez Fernández, M., Fragoso Campón, L., Gutiérrez Gallego J. A., Quirós Rosado E. (2017): "Caída de la demanda con la distancia en el uso del transporte público: el caso del acceso al campus universitario de Cáceres", GeoFocus (Artículos), $n^{o}$ 20, p. 141-163. ISSN: 1578-5157 http://dx.doi.org/10.21138/GF.563

De los dos modelos analizados podemos observar cómo, para el caso del Escenario 2, el ratio de viajes por habitante obtenido mediante la fórmula de Tanner se ajusta mejor a la densidad de las encuestas realizadas, representando un ajuste espacial más acorde con el comportamiento reflejado en las encuestas de los usuarios. En este escenario, en el que el usuario se desplaza hasta una de las dos paradas principales, se observa cómo el peatón recorrerá una mayor distancia dependiendo de la situación de la vivienda respecto a las distintas paradas existentes a lo largo de la línea de autobús. La Figura 10 muestra la distancia recorrida por el usuario en el caso de desplazarse hacia la parada principal más cercana a su domicilio, en vez de seleccionar la parada más próxima al mismo.

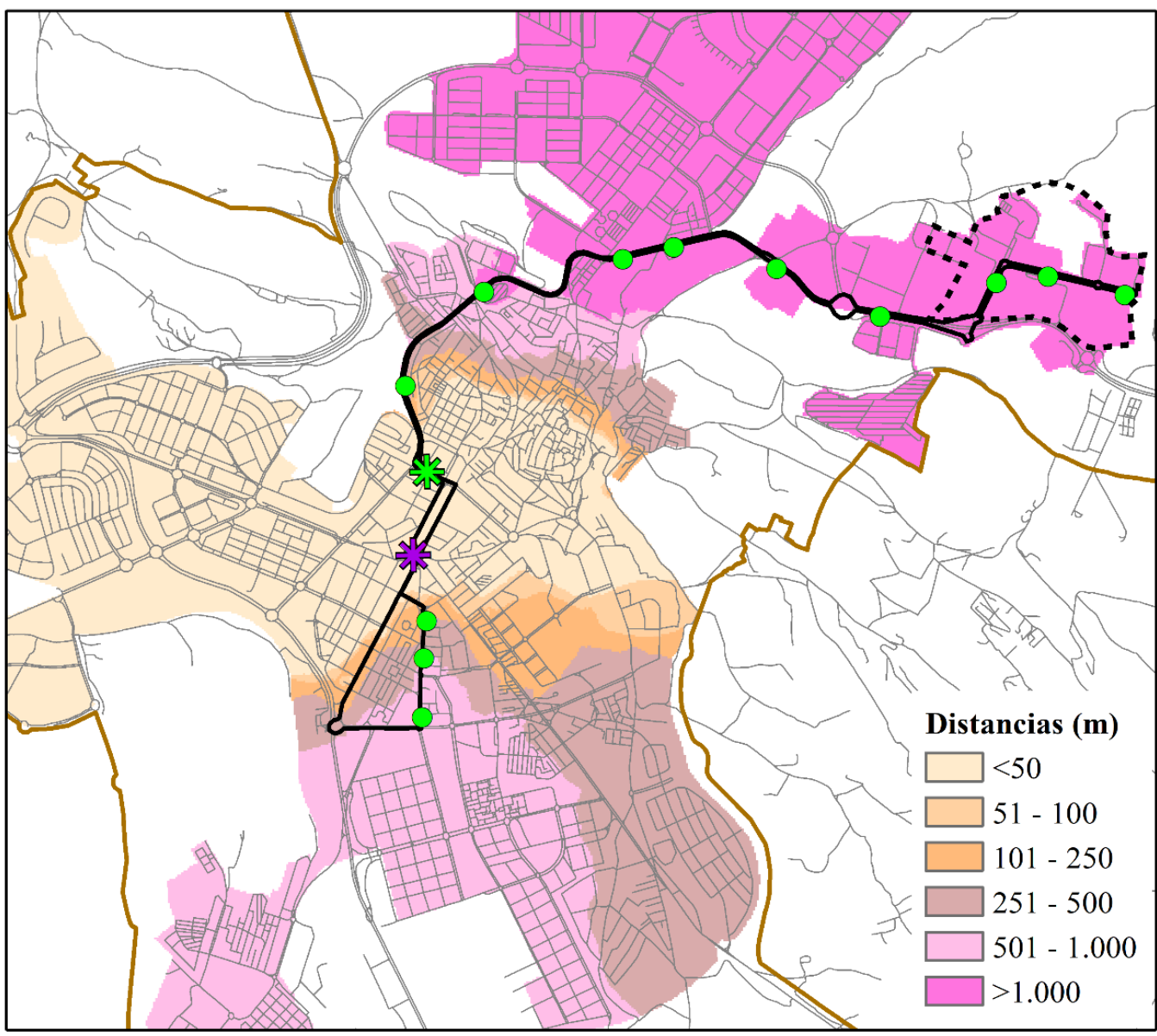

Leyenda

-.-. Campus Universitario Paradas Principales

Parada de autobús

— Línea de autobús Nis Avda. General Primo de Rivera

Resto de paradas

Contorno urbano

龂 Plaza de América

Eje de viales

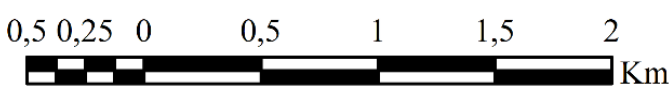

Figura 10. Incremento de la distancia recorrida por el usuario en el caso de desplazarse hacia la parada principal más cercana a su domicilio, en vez de seleccionar la parada más próxima al mismo. 
Sánchez Fernández, M., Fragoso Campón, L., Gutiérrez Gallego J. A., Quirós Rosado E. (2017): "Caída de la demanda con la distancia en el uso del transporte público: el caso del acceso al campus universitario de Cáceres", GeoFocus (Artículos), $n^{\circ}$ 20, p. 141-163. ISSN: 1578-5157 http://dx.doi.org/10.21138/GF.563

\section{Discusión}

Los resultados obtenidos en la caracterización del usuario muestran una centralización de la demanda del servicio tan sólo en dos de las 12-15 paradas (según la línea de autobús) que el autobús tiene a lo largo del recorrido, a las que se ha denominado paradas principales ("Plaza de América" y "Avda. General Primo de Rivera") y que acogen al $82 \%$ de los usuarios encuestados. Este aspecto está en concordancia con los señalado en Carpio-Pinedo (2014), ya que la elección más probable de la parada de autobús, no solo está influida por la distancia más corta, sino por otras variables que intervienen en la decisión final del peatón a la hora de elegir la parada. En este caso, al ser Cáceres una ciudad de mediano tamaño, el viajero está dispuesto a caminar una mayor distancia hasta una de las dos paradas principales, con el objetivo de garantizarse el acceso al autobús ya que, debido a la alta demanda del servicio, sobre todo en hora punta, una vez que el autobús presenta aforo completo, ya no realiza parada en el resto de los puntos de acceso al mismo. Observándose cómo en ciertas zonas de la ciudad, que aun teniendo una parada más cercana al domicilio, los usuarios prefieren recorrer una mayor distancia para acceder a las paradas principales.

La estimación de la función de la caída de la demanda con la distancia mediante la fórmula de Tanner ofrece un ajuste adecuado a los datos empíricos, incluso para los intervalos de distancias más cortas, al contrario que en los resultados expuestos por Martínez y Viegas (2013), en los que la fórmula de Tanner no se ajustaba correctamente en distancias cortas (tiempo de recorrido inferior a 3 minutos: aproximadamente a unos $200 \mathrm{~m}$ según una velocidad de peatón media de 4,5 km/h (Munoz-Raskin 2010)). Los resultados obtenidos en el escenario más realista de los dos estudiados, en el que el peatón se dirige hacia una de las dos paradas principales, muestran como el ratio de viajes/población, que representa la probabilidad de uso del servicio, disminuye bruscamente a partir de los 900-1.300 m, presentando los mejores resultados para una distancia inferior a $\operatorname{los} 600 \mathrm{~m}$. Este sería por tanto el área de influencia de las dos paradas principales de las líneas de autobús que dan acceso al campus, y se observa como están en concordancia con las distancias obtenidas por otros autores a nivel nacional, como el intervalo entre 300 y $600 \mathrm{~m}$ definido por Gutiérrez Puebla et al. (2002), y el valor de $800 \mathrm{~m}$ fijado por Cardozo et al. (2010). A nivel internacional el resultado obtenido es similar al caso de El-Geneidy et al. (2014), que obtenía una distancia límite del orden de $524 \mathrm{~m}$ para el acceso al transporte en autobús en su estudio en Montreal.

\section{Conclusiones}

En este trabajo se ha estudiado el potencial uso del transporte público como modo de acceso al campus universitario de Cáceres, atendiendo a la cercanía del domicilio de los usuarios a las paradas de autobús. Gracias a la integración de los SIG con otras herramientas informáticas, como los paquetes estadísticos y ofimáticas, ha sido posible alcanzar con suficiencia los objetivos propuestos. Sin necesidad de recurrir a aplicaciones específicas para el diseño y evaluación de modelos de transporte.

Los resultados obtenidos de las encuestas han permitido realizar una caracterización del usuario del autobús dentro de la comunidad universitaria de Cáceres, tratándose probablemente 
Sánchez Fernández, M., Fragoso Campón, L., Gutiérrez Gallego J. A., Quirós Rosado E. (2017): "Caída de la demanda con la distancia en el uso del transporte público: el caso del acceso al campus universitario de Cáceres", GeoFocus (Artículos), $n^{o}$ 20, p. 141-163. ISSN: 1578-5157 http://dx.doi.org/10.21138/GF.563

de una mujer menor de 25 años, estudiante de grado y que utiliza una de las dos paradas principales como origen del trayecto.

Se ha observado como la elección más probable de la parada de autobús no solo está influida por la distancia más corta, sino por otras variables que intervienen en la decisión final del usuario a la hora de elegir la parada de autobús. En este caso el viajero está dispuesto a caminar una mayor distancia hasta una de las dos paradas principales, con el objetivo de garantizarse el acceso al autobús.

De los dos escenarios analizados para determinar la caída de la demanda con la distancia, el basado en que el usuario accede a la parada más cercana, se ha determinado una distancia límite de uso de 600 metros. Mientras que en el segundo escenario, en el que el usuario accede a una de las paradas principales, el radio de influencia se eleva a 1.300 metros.

A partir de este trabajo sería posible estudiar medidas de mejora y optimización de la explotación del transporte público. Se podrían plantear otras alternativas de trayecto o incluso la incorporación de nuevas líneas para aumentar la utilización del servicio de autobús urbano que da acceso al campus universitario y así fomentar más la utilización del transporte público como alternativa al vehículo privado.

\section{Agradecimientos}

A la empresa SUBUS por haber facilitado los datos para la elaboración de este estudio.

A los compañeros y profesores de la asignatura Iniciación a la Investigación en Expresión Gráfica en Ingeniería del MUI en Ingeniería y Arquitectura - Expresión Gráfica y Construcción, curso académico 2014/15, por haber colaborado en el proyecto y en la toma de encuestas.

\section{Referencias bibliográficas}

Alfonso-Torreño, A. (2013):"Situación de las barriadas de Cáceres respecto al campus universitario: Análisis de la movilidad obligada". Los servicios: Dinámicas, infraestructuras y cohesión territorial 2013, 483-500.

Awad, S., 2015. La movilidad sostenible: un reto de las ciudades en el s.XXI.

Berrocal Nieto, R. y Ruiz Labrador, E. E. (2013):"Metodología para la determinación de la accesibilidad peatonal a la red de transporte urbano de Mérida". Los servicios: Dinámicas, infraestructuras y cohesión territorial 2013, 501-518.

Campesino Fernández, A. J. y Salcedo Hernández, J. C. (2007): "Campus universitarios en ciudades patrimoniales: contrastes entre Cáceres y Toledo". CIAN-Revista de Historia de las Universidades, 17, 1, 101-137.

Cardozo, O. D., Gutiérrez Puebla, J. y García Palomares, J. C. (2010): "Influencia de la morfología urbana en la demanda de transporte público: análisis mediante SIG y modelos de regresión múltiple". GeoFocus, 10, 82-102.

Carpio-Pinedo, J. (2014): "Urban bus demand forecast at stop level: Space Syntax and other built environment factors. Evidence from Madrid". Procedia - Social and Behavioral Sciences, 160, 205-214. 
Sánchez Fernández, M., Fragoso Campón, L., Gutiérrez Gallego J. A., Quirós Rosado E. (2017): "Caída de la demanda con la distancia en el uso del transporte público: el caso del acceso al campus universitario de Cáceres", GeoFocus (Artículos), $n^{\circ}$ 20, p. 141-163. ISSN: 1578-5157 http://dx.doi.org/10.21138/GF.563

Cartociudad (2015): Directorio de Servicios de CartoCiudad. Gobierno de España. http://www.cartociudad.es/portal/ (consultado 01-06-2015).

Cochran, W. G. (1990): Técnicas de muestreo. CECSA, México.

Díaz Muñoz, M. Á., et al. (2007): "Propuesta de un sistema de indicadores de sostenibilidad para la movilidad y el transporte urbanos. Aplicación mediante SIG a la ciudad de Alcalá de Henares". Cuadernos de Geografía, 81-82, 31-50.

El-Geneidy, A., et al. (2014): "New evidence on walking distances to transit stops: Identifying redundancies and gaps using variable service areas". Transportation, 41, 1, 193-210.

García-Palomares, J. C., Gutiérrez, J. y Cardozo, O. D. (2013): "Walking accessibility to public transport: an analysis based on microdata and GIS". Environment and Planning B: Planning and Design, 40, 6, 1087-1102.

García Palomares, J. C. y Gutiérrez Puebla, J. (2007): "Pautas de la movilidad en el área metropolitana de Madrid". Cuadernos de Geografía, 81-82, 7-29.

Gutiérrez Gallego, J. A. y Jaraíz Cabanillas, F. J., 2013. Aproximación al Plan de Movilidad Sostenible del Campus Universitario de Cáceres de la UEX. In: EMPRESA, G. D. R. V. E. L. ed. Ciclo de jornadas sobre prevención de riesgos laborales. Universidad de Extremadura: Universidad de Extremadura

Gutiérrez, J., Condeço-Melhorado, A. y Martín, J. C. (2010): "Using accessibility indicators and GIS to assess spatial spillovers of transport infrastructure investment". Journal of Transport Geography, 18, 1, 141-152.

Gutiérrez Puebla, J., Cardozo, O. D. y García Palomares, J. C. (2008):"Modelos de demanda potencial de viajeros en redes de transporte público: aplicaciones en el Metro de Madrid". IV Seminario de Ordenación Territorial 2008.

Gutiérrez Puebla, J., et al. (2002):"Accesibilidad peatonal a la red sanitaria de asistencia primaria en Madrid". Anales de Geografía de la Universidad Complutense 2002, 269280.

Ine (2015): Instituto Nacional de Estadística. INE. http://www.ine.es (consultado 01-06-2015).

Jaraíz Cabanillas, F. J., Naranjo Gómez, J. M. y Marrodán Castro, S. (2015):"Modelos de demanda potencial en función del tiempo de acceso a los servicios: el caso del aeropuerto de Badajoz". VII Congreso de geografía de los servicios: El papel de los servicios en la construcción del territorio: redes y actores. 11-13 de junio 2015 Alicante, 447-472.

Jaraíz Cabanillas, F. J., Ruiz Labrador, E. E. y Gutiérrez Gallego, J. A. (2013):"Análisis de la movilidad en el acceso a los centros educativos. El caso de Mérida". Los servicios: Dinámicas, infraestructuras y cohesión territorial 2013, 519-534.

Jensen, S. U. (2008): "How to obtain a healthy journey to school". Transportation research part A: policy and practice, 42, 3, 475-486.

Martínez, L. M. y Viegas, J. M. (2013): "A new approach to modelling distance-decay functions for accessibility assessment in transport studies". Journal of Transport Geography, 26, 87-96.

Mora Aliseda, J., et al. (2003): "Aplicación de técnicas de SIG en la planificación del transporte en Extremadura (España)". Finisterra, 38, 75, 67-83.

Munoz-Raskin, R. (2010): "Walking accessibility to bus rapid transit: Does it affect property values? The case of Bogotá, Colombia". Transport Policy, 17, 2, 72-84.

Muñoz, F. (2006): El tiempo del territorio, los territorios del tiempo. Las otras geografías. Tirant lo Blanch, 235-254.

Murray, A. T. (2001): "Strategic analysis of public transport coverage". Socio-Economic Planning Sciences, 35, 3, 175-188. 
Sánchez Fernández, M., Fragoso Campón, L., Gutiérrez Gallego J. A., Quirós Rosado E. (2017): "Caída de la demanda con la distancia en el uso del transporte público: el caso del acceso al campus universitario de Cáceres", GeoFocus (Artículos), $n^{\circ}$ 20, p. 141-163. ISSN: 1578-5157 http://dx.doi.org/10.21138/GF.563

Navarro Grueso, F. M. (2013):"Propuesta metodológica para el análisis del estacionamiento para la mejora de la movilidad sostenible. El caso del campus universitario de Cáceres". Los servicios: Dinámicas, infraestructuras y cohesión territorial 2013, 553-570.

Obin (2015): Observatorio de Indicadores de la Universidad de Extremadura. Universidad de Extremadura. http://www.unex.es/organizacion/serviciosuniversitarios/unidades/utec/funciones/estadisticas-e-indicadores-universitarios (consultado 01-06-2015).

Ortuzar, J. D. D. y Willumsen, L. G. (2008): Modelos de transporte. Ed. Universidad de Cantabria.

Pitarch Garrido, M. D. (2015):"Polarización social y territorial: la localización de la oferta de servicios públicos para el análisis de la equidad territorial. Aplicación al caso del área metropolitana de Valencia". VII Congreso de geografía de los servicios: El papel de los servicios en la construcción del territorio: redes y actores. 11-13 de junio 2015 Alicante, 303-324.

Quinto Bernabeu, F. J. (2015):"Aproximación al área de captación de viajeros y su repercusión comercial en la parada de metro ligero de San Vicente del Raspeig (Alicante)". VII Congreso de geografía de los servicios: El papel de los servicios en la construcción del territorio: redes y actores. 11-13 de junio 2015 Alicante, 473-490.

Rodríguez Díaz, V. (2011): "Medición de la accesibilidad geográfica de la población a los Hospitales de Alta Resolución de Andalucía mediante herramientas SIG basadas en el análisis de redes". GeoFocus. Revista Internacional de Ciencia y Tecnología de la Información Geográfica, 11, 265-292.

Sig_Cáceres (2015): Servicio de Sistema de Información Geográfica de Cáceres. http://sig.caceres.es/ (consultado 01-06-2015).

Team, R. C., 2016. R: A language and environment for statistical computing. R Foundation for Statistical Computing, Vienna, Austria.

Tiefelsdorf, M. (2003): "Misspecifications in interaction model distance decay relations: A spatial structure effect". Journal of Geographical Systems, 5, 1, 25-50.

Zhao, F., et al. (2003): "Forecasting transit walk accessibility: regression model alternative to buffer method". Transportation Research Record: Journal of the Transportation Research Board, 1835, 34-41. 
